Research Article

\title{
Serum-Free Thyroxine Levels Were Associated with Pulmonary Hypertension and Pulmonary Artery Systolic Pressure in Euthyroid Patients with Coronary Artery Disease
}

\author{
Bingjie Wu, Jingjing Jiang, Minghui Gui, Lin Liu, Qiqige Aleteng, Shanshan Wang, \\ Xiaojing Liu, Yan Ling, and Xin Gao \\ Department of Endocrinology and Metabolism, Zhongshan Hospital, Fudan University, No. 180 Fenglin Road, \\ Shanghai 200032, China \\ Correspondence should be addressed to Yan Ling; doctorlingyan@163.com and Xin Gao; zhongshan_endo@126.com
}

Received 16 August 2016; Revised 19 April 2017; Accepted 24 April 2017; Published 22 June 2017

Academic Editor: Carlo Cappelli

Copyright (c) 2017 Bingjie Wu et al. This is an open access article distributed under the Creative Commons Attribution License, which permits unrestricted use, distribution, and reproduction in any medium, provided the original work is properly cited.

\begin{abstract}
The aim of this study was to evaluate the association between thyroid hormone levels, pulmonary hypertension (PH), and pulmonary artery systolic pressure (PASP) in euthyroid patients with coronary artery disease (CAD). A cross-sectional study was conducted in individuals who underwent coronary angiography and were diagnosed as CAD from March 2013 to November 2013. 811 subjects ( 185 women and 626 men) were included in this study. PASP was measured by transthoracic Doppler echocardiography. 86 patients were diagnosed as $\mathrm{PH}$ and had significantly higher free thyroxine $\left(\mathrm{FT}_{4}\right)$ levels than those without PH. Multiple logistic regression analysis demonstrated an independent association of $\mathrm{FT}_{4}$ levels with $\mathrm{PH}$ after adjustment of gender, age, body mass index, systolic blood pressure, left ventricular ejection fraction, hypertension, and medication use of calcium channel blockers, ACE inhibitors, angiotensin II receptor antagonists, and nitrates. Serum-free triiodothyronine $\left(\mathrm{FT}_{3}\right)$ and thyroid-stimulating hormone $(\mathrm{TSH})$ were not associated with $\mathrm{PH}$. Furthermore, multivariate linear regression analysis showed that $\mathrm{FT}_{4}$ levels emerged as an independent predictor for PASP, while $\mathrm{FT}_{3}$ and TSH levels were not associated with PASP. Our study demonstrated that, in euthyroid patients with $\mathrm{CAD}, \mathrm{FT}_{4}$ was an independent risk factor for $\mathrm{PH}$, and $\mathrm{FT}_{4}$ levels were independently associated with PASP.
\end{abstract}

\section{Introduction}

Pulmonary hypertension $(\mathrm{PH})$ is a hemodynamic and pathological state with a high rate of mortality that may lead to right heart failure and ultimately death if untreated. $\mathrm{PH}$ can be found in multiple clinical conditions and its symptoms are nonspecific. Badesch et al. [1] brought attention to the relationship between $\mathrm{PH}$ and hypothyroidism and growing evidences confirmed the association between $\mathrm{PH}$ and thyroid diseases thereafter. Curnock et al. [2] revealed in their retrospective study that the prevalence of hypothyroidism in 41 patients with $\mathrm{PH}$ was $22.5 \%$. Chu et al. [3] found that the prevalence of autoimmune thyroid disease in the patients with $\mathrm{PH}$ was $49 \%$. In another study, the prevalence of thyroid disease was $24 \%$ in $\mathrm{PH}$ patients and $15 \%$ in the control group [4]. On the other hand, patients with thyroid disease, predominantly hyperthyroidism, also have higher pulmonary arterial pressure than healthy subjects. The prevalence of $\mathrm{PH}$ among patients with hyperthyroidism was reported to be 34-65\% [5-9], and pulmonary artery systolic pressure (PASP) decreased after treatment of hyperthyroidism.

These results aforementioned suggested a close relationship between thyroid diseases and PH. Thyroid hormones may play an important role in regulating PASP. However, the association of thyroid hormones with $\mathrm{PH}$ remains controversial. Marvisi et al. [10] demonstrated that in population with recently diagnosed hyperthyroidism without antithyroid treatment, PASP was associated 
with thyroid-stimulating hormone (TSH) and free thyroxine $\left(\mathrm{FT}_{4}\right)$ levels. However, Sugiura et al. [11] showed that PASP was not significantly correlated with free triiodothyronine $\left(\mathrm{FT}_{3}\right)$ or $\mathrm{FT}_{4}$ in patients with Graves' disease. And in patients with Hashimoto's thyroiditis, it was shown that $\mathrm{FT}_{3}, \mathrm{FT}_{4}$, or TSH was not independently related to PASP $[12,13]$.

Meanwhile, PASP is considered as an important prognostic factor for assessing morbidity and mortality in patients with CAD [14-16]; thus, it is essential to find out the potential risk factors for $\mathrm{PH}$ in $\mathrm{CAD}$ patients. According to previous studies, it is acknowledged that thyroid dysfunction could induce $\mathrm{PH}$ via multiple pathways, but whether thyroid hormones within reference range would affect PASP is still uncertain. To the best of our knowledge, the association between thyroid hormones and $\mathrm{PH}$ was never examined in CAD subjects. To address this issue, we sought to clarify the possible relationship between thyroid hormones, PH, and PASP in euthyroid patients with CAD.

\section{Study Population and Methods}

During March 2013 to November 2013, we consecutively enrolled 2045 patients who were admitted to the Department of Cardiology of Zhongshan Hospital for suspected CAD and underwent coronary angiography. Data of participants were collected by a structured interview and medical record review. Smoking, history of hypertension, heart failure, chronic obstructive pulmonary disease, and medication use (calcium channel blockers, ACE inhibitors, angiotensin II receptor antagonists, and nitrate drugs) were recorded. Coronary angiography was performed by experienced doctors using a digital angiography system (AXIOM Artis dFC, Siemens, Germany). Coronary artery branches including the left coronary artery, left anterior descending artery, left circumflex artery, left marginal artery, diagonal branch, right coronary artery, posterior descending artery, and right marginal artery were evaluated. A luminal stenosis of $50 \%$ or more of any branch was defined as CAD.

Exclusion criteria were listed as follows: history of heart failure, chronic obstructive pulmonary disease, hypothyroidism, hyperthyroidism, severe systemic diseases, malignancy, using medications (antithyroid medications, thyroid hormone, amiodarone, and glucocorticoid hormone) influencing thyroid function, luminal stenosis of all branches less than $50 \%$ measured by coronary angiography, and patients with uncompleted data. Finally, 811 subjects (185 women and 626 men) were included in the analyses. The study was approved by the Ethical Committee of Zhongshan Hospital affiliated to Fudan University. Informed consent was obtained from each participant.

Anthropometric parameters were collected for each individual. Weight and height were measured and body mass index (BMI) was calculated as weight divided by height squared $\left(\mathrm{kg} / \mathrm{m}^{2}\right)$. Blood samples were obtained before angiography. Serum $\mathrm{FT}_{3}, \mathrm{FT}_{4}$, and $\mathrm{TSH}$ were measured by a model 7600 automated bioanalyzer (Hitachi, Tokyo, Japan). Hypothyroidism was defined as TSH $>4.2 \mathrm{mU} / \mathrm{L}$ with or without decreased thyroid hormones, and hyperthyroidism was defined as $\mathrm{TSH}<0.27 \mathrm{mU} / \mathrm{L}$ with or without elevated thyroid hormones.

Transthoracic Doppler echocardiogram was performed using an iE33 Echocardiograph (Philips Medical Systems, Bothell, WA, USA). Two-dimensional images were obtained. The end-diastolic volume (EDV) and endsystolic volume (ESV) were measured, then left ventricular ejection fraction (LVEF) was calculated as follow: $\operatorname{LVEF}(\%)=(\mathrm{EDV}-\mathrm{ESV}) / \mathrm{EDV} \times 100 \%$. The tricuspid regurgitation peak velocity (TRPV) and right atrial pressure (RAP) were recorded, and PASP was calculated using the formula: PASP $=4 \times \mathrm{TRPV}+\mathrm{RAP}$. In addition, 20 participants were selected randomly to assess the interobserver and intraobserver variability. Two experienced echocardiographers performed the exact same procedure of echocardiogram to determine the interobserver variability. One of the echocardiographers repeated the procedure the next day to determine the intraobserver variability. The interobserver variability coefficients were $5.1 \%, 4.5 \%$, 5.6\%, 6.1\%, 5.9\%, and 7.9\% for EDV, ESV, LVEF, TRPV, RAP, and PASP, respectively. The intraobserver variability coefficients were $4.2 \%, 4.3 \%, 5.0 \%, 4.9 \%, 5.7 \%$, and $7.0 \%$ for EDV, ESV, LVEF, TRPV, RAP, and PASP, respectively. $\mathrm{PH}$ was defined as PASP $>40 \mathrm{mmHg}$ [17].

\section{Statistical Analyses}

Continuous variables were reported as mean \pm standard deviation, while categorical variables were represented by frequency and percentage. To determine the potential factors influencing $\mathrm{PH}$, participants were stratified according to the values of PASP, and the stratifications were defined as follows: non-PH: PASP $\leq 40 \mathrm{mmHg}$, mild $\mathrm{PH}$ : $40 \mathrm{mmHg}$ $<\mathrm{PASP} \leq 50 \mathrm{mmHg}$, and moderate-to-severe $\mathrm{PH}: \mathrm{PASP}>$ $50 \mathrm{mmHg}$ [18]. On the other hand, in order to compare the differences of PASP levels between different thyroid functions, the study subjects were also stratified according to the tertile groups of thyroid hormones. $\mathrm{FT}_{3}$ tertiles are divided as follows: tertile 1: $\mathrm{FT}_{3} \leq 4.1(n=308)$, tertile 2: $4.1<\mathrm{FT}_{3} \leq 4.5(n=236)$, and tertile $3: \mathrm{FT}_{3}>4.5(n=267)$; $\mathrm{FT}_{4}$ tertiles: tertile 1: $\mathrm{FT}_{4} \leq 14.2 \quad(n=260)$, tertile 2: $14.2<\mathrm{FT}_{4} \leq 16.1(n=279)$, tertile $3: \mathrm{FT}_{4}>16.1(n=272)$; and TSH tertiles: tertile 1: TSH $\leq 1.57 \quad(n=275)$, tertile 2: $1.57<\mathrm{TSH} \leq 2.59(n=277)$, and tertile $3: \mathrm{TSH}>2.59$ $(n=259)$. One-way ANOVA analysis and analysis of covariance were used for comparisons for continuous variables, and chi-square tests were performed for categorical variables among the groups. Logistic regression analysis was performed to determine the independent risk factors for $\mathrm{PH}$. Multivariate linear regression analysis was used to determine independent risk factors influencing PASP. All statistical analyses were performed using the SPSS statistics version 17.0 (SPSS Inc., Chicago, IL). All tests were two tailed, and $P<0.05$ was considered statistically significant.

\section{Results}

The mean age of the study subjects was $63.3 \pm 9.87$ years, and the mean PASP was $34 \pm 8 \mathrm{mmHg}$. The study population was 
divided into two groups according to PASP measured by echocardiography: 86 suffering from PH and 725 had normal PASP. Table 1 shows the baseline clinical characteristics of each group. Compared with those with the non- $\mathrm{PH}$ group, patients with $\mathrm{PH}$ were older. There were more smokers in the $\mathrm{PH}$ group compared to those in the non- $\mathrm{PH}$ group $(P<0.05)$. The $\mathrm{PH}$ group had significantly higher levels of $\mathrm{FT}_{4}(P<0.05)$ and lower LVEF $(P<0.05)$ than those of the non-PH group. Serum $\mathrm{FT}_{3}$ and TSH did not differ significantly between the two groups.

Furthermore, we compared the thyroid hormone levels among subgroups with different PASP (Table 2). An increase of serum $\mathrm{FT}_{4}$ levels was found as PASP increased. $\mathrm{FT}_{4}$ levels of the mild $\mathrm{PH}$ and moderate-to-severe $\mathrm{PH}$ groups were higher than those in the non-PH group $(15.75 \pm 2.31$ and $16.80 \pm 2.94$ versus $15.30 \pm 2.07 \mathrm{mmol} / \mathrm{L}$, both $P<0.05)$. Serum TSH and $\mathrm{FT}_{3}$ levels were not significantly different among subgroups with different PASP. Moreover, the study subjects were further stratified according to thyroid hormones tertiles (Table 3). In the $\mathrm{FT}_{3}$ tertile groups, PASP were higher in the first tertile than in the other two tertiles $(P<0.01)$. After adjusted for age, gender, BMI, and LVEF, the significant difference disappeared $(P=0.06)$. No significant difference of PASP was found among the $\mathrm{FT}_{4}$ and TSH tertile groups $(P=0.42$ and $P=0.36$, resp.).

To determine the relationship between $\mathrm{PH}$ and thyroid hormones, multiple logistic regression analyses were performed (Table 4). After adjustment of age, gender, BMI, smoking, LVEF, hypertension, and use of calcium channel blockers, ACE inhibitors, angiotensin II receptor antagonists, and nitrates, $\mathrm{FT}_{4}$ levels were independently associated with the risk of $\mathrm{PH}(\mathrm{OR}=1.12,95 \% \mathrm{CI} 1.01-1.25, P=0.03)$. However, levels of serum $\mathrm{FT}_{3}$ and TSH were not independently associated with $\mathrm{PH}(\mathrm{OR}=1.18,95 \% \mathrm{CI} 0.79-1.82, P=0.45$ and $\mathrm{OR}=0.97,95 \% \mathrm{CI} 0.84-1.12, P=0.70$, resp.).

Multivariate linear regression analyses were performed to further analyze the risk factors influencing PASP (Table 5). Serum $\mathrm{FT}_{4}$ levels $(\beta=0.27 \pm 0.12, \quad P=0.03)$ and age $(\beta=0.11 \pm 0.03, P<0.01)$ were positively associated with PASP adjusted for age, gender, BMI, smoking, LVEF, hypertension, and use of calcium channel blockers, ACE inhibitors, angiotensin II receptor antagonists, and nitrates. And a negative association was observed between LVEF and PASP $(-0.13 \pm 0.03, P<0.01)$. In contrast, levels of $\mathrm{FT}_{3}(\beta=-0.53$ $\pm 0.49, P=0.28)$ and TSH $(\beta=0.00 \pm 0.15, P=0.99)$ were not significantly associated with PASP.

\section{Discussion}

In the present study, we investigated the relationship between thyroid function and PASP in euthyroid patients with $\mathrm{CAD}$. We observed that serum $\mathrm{FT}_{4}$ levels were not only significantly higher in patients with $\mathrm{PH}$ than patients without $\mathrm{PH}$ but also independently associated with $\mathrm{PH}$ and PASP. In contrast, serum $\mathrm{FT}_{3}$ or TSH levels were not associated with $\mathrm{PH}$ or PASP. To the best of our knowledge, the present study is the first to explore the independent relationship between thyroid hormones and $\mathrm{PH}$ in euthyroid patients with CAD.
TABLE 1: Characteristics of study participants.

\begin{tabular}{|c|c|c|c|}
\hline & $\mathrm{PH}(n=86)$ & $\begin{array}{l}\text { Non-PH } \\
(n=725)\end{array}$ & $P$ value \\
\hline Male (\%) & 69.8 & 78.1 & 0.10 \\
\hline PASP (mmHg) & $49 \pm 11$ & $32 \pm 4$ & $<0.01$ \\
\hline Age (years) & $68.27 \pm 9.42$ & $62.74 \pm 9.77$ & $<0.01$ \\
\hline BMI $\left(\mathrm{kg} / \mathrm{m}^{2}\right)$ & $24.63 \pm 3.19$ & $24.63 \pm 2.97$ & 0.99 \\
\hline SBP (mmHg) & $129.10 \pm 12.25$ & $129.02 \pm 13.81$ & 0.97 \\
\hline DBP (mmHg) & $77.05 \pm 8.23$ & $77.42 \pm 8.37$ & 0.69 \\
\hline $\mathrm{FT}_{4}(\mathrm{mmol} / \mathrm{L})$ & $16.07 \pm 2.55$ & $15.30 \pm 2.08$ & $<0.01$ \\
\hline $\mathrm{FT}_{3}(\mathrm{mmol} / \mathrm{L})$ & $4.25 \pm 0.70$ & $4.32 \pm 0.56$ & 0.35 \\
\hline $\mathrm{TSH}(\mu \mathrm{IU} / \mathrm{ml})$ & $2.43 \pm 1.69$ & $2.40 \pm 1.74$ & 0.92 \\
\hline LVEF (\%) & $58 \pm 14$ & $63 \pm 9$ & $<0.01$ \\
\hline Hypertension (\%) & 75.6 & 65.0 & 0.05 \\
\hline History of smoking (\%) & 16.3 & 7.1 & 0.01 \\
\hline ACEI \& ARB (\%) & 54.7 & 47.1 & 0.21 \\
\hline Nitrates (\%) & 40.7 & 35.9 & 0.41 \\
\hline CCB (\%) & 26.7 & 26.3 & 1.00 \\
\hline
\end{tabular}

PH: pulmonary hypertension; PASP: pulmonary artery systolic pressure; BMI: body mass index; SBP: systolic blood pressure; $\mathrm{FT}_{4}$ : free thyroxine; $\mathrm{FT}_{3}$ : free triiodothyronine; TSH: thyroid-stimulating hormone; LVEF: left ventricular ejection fraction; CCB: calcium channel blockers; ACEI \& ARB: ACE inhibitors and angiotensin II receptor antagonists. Data are presented as the mean \pm standard deviation or as percentages.

CAD is highly prevalent worldwide and has become one of the top causes of death. Left heart diseases have been considered as a common cause of PH. It may lead to an increase in left atrial pressure, and subsequently, pulmonary artery pressure increases due to the passive backward transmission [19]. Meanwhile, $\mathrm{PH}$ is potential to alter right ventricular afterload, resulting in increasing cardiac output and may lead to left ventricle dysfunction [14]. A retrospective analysis performed in 6611 patients with CAD undergoing bilateral heart catheterization indicated that $61.3 \%$ had abnormal right heart catheterization values and $4.3 \%$ met formal criteria for $\mathrm{PH}$ [20]. It was also reported that secondary $\mathrm{PH}$ predicted mortality in patients with acute myocardial infarction [15]. The tricuspid regurgitation and end-diastolic pulmonary regurgitation gradients, in conjunction with PASP, have been emerged as a potential predictor of mortality and heart failure hospitalization in patients with stable coronary artery disease [16]. In this setting, it is essential to determine the possible risk factors for PASP in CAD. On the other hand, several studies demonstrated that levels of $\mathrm{FT}_{4}$ were independently associated with increased prevalence of coronary calcification and $\mathrm{FT}_{3}$ levels were independent predictors for both the presence and severity of CAD in euthyroid population [21-23]. However, whether thyroid hormone levels could be a factor for increasing PASP which predicts prognosis of patients with CAD is still uncertain.

Growing evidences suggested that thyroid disease is closely related to $\mathrm{PH}$. Patients with thyroid disease had relatively high PASP values [5-9], while PH patients had high prevalence of thyroid diseases [1-4]. A number of prospective studies found that increase of PASP in hyperthyroidism 
TABLE 2: Comparison of thyroid function parameters among subgroups with different pulmonary artery systolic pressures.

\begin{tabular}{lccccc}
\hline & Non-PH & Mild PH & Moderate-to-severe & Model 1 & Model 2 \\
$P$ value & PH $(n=26)$ & 0.40 & $P$ value \\
\hline $\mathrm{FT}_{3}$ & $4.32 \pm 0.56$ & $4.28 \pm 0.68$ & $4.18 \pm 0.74$ & 0.38 \\
$\mathrm{FT}_{4}$ & $15.30 \pm 2.07$ & $15.75 \pm 2.31^{*}$ & $16.80 \pm 2.94^{* \#}$ & $<0.01$ \\
$\mathrm{TSH}$ & $2.41 \pm 1.74$ & $2.38 \pm 1.78$ & $2.53 \pm 1.48$ & 0.93 & 0.01 \\
\hline
\end{tabular}

PH: pulmonary hypertension; $\mathrm{FT}_{4}$ : free thyroxine; $\mathrm{FT}_{3}$ : free triiodothyronine; $\mathrm{TSH}$ : thyroid-stimulating hormone; Model 1: unadjusted; Model 2: adjusted for age, gender, BMI, and LVEF. Data are presented as the mean \pm standard deviation. ${ }^{*} P<0.05$ versus the non-PH group, ${ }^{\#} P<0.05$ versus the mild $\mathrm{PH}$ group.

TABLE 3: Comparison of pulmonary artery systolic pressure among the tertile groups of thyroid function parameters.

\begin{tabular}{lccccc}
\hline & Tertile 1 & Tertile 2 & Tertile 3 & $\begin{array}{l}\text { Model 1 } \\
P \text { value }\end{array}$ & $\begin{array}{l}\text { Model 2 } \\
P \text { value }\end{array}$ \\
\hline $\mathrm{FT}_{3}$ & $34.75 \pm 8.13$ & $32.71 \pm 5.99$ & $33.57 \pm 7.50$ & $<0.01$ & 0.06 \\
$\mathrm{FT}_{4}$ & $33.10 \pm 5.81$ & $33.29 \pm 6.97$ & $34.31 \pm 9.23$ & 0.14 & 0.42 \\
$\mathrm{TSH}$ & $33.05 \pm 6.60$ & $34.13 \pm 8.74$ & $33.53 \pm 6.94$ & 0.24 & 0.36 \\
\hline
\end{tabular}

$\mathrm{FT}_{4}$ : free thyroxine; $\mathrm{FT}_{3}$ : free triiodothyronine; $\mathrm{TSH}$ : thyroid-stimulating hormone; Model 1: unadjusted; Model 2: adjusted for age, gender, BMI, and LVEF. $\mathrm{FT}_{3}$ tertiles were divided as follows: tertile $1: \mathrm{FT}_{3} \leq 4.1 \quad(n=308)$, tertile 2: $4.1<\mathrm{FT}_{3} \leq 4.5(n=236)$, and tertile 3: $\mathrm{FT}_{3}>4.5(n=267) . \mathrm{FT}_{4}$ tertiles were divided as follows: tertile $1: \mathrm{FT}_{4} \leq 14.2(n=260)$, tertile 2 : $14.2<\mathrm{FT}_{4} \leq 16.1(n=279)$, and tertile $3: \mathrm{FT}_{4}>16.1(n=272)$. TSH tertiles were divided as follows: tertile 1: TSH $\leq 1.57 \quad(n=275)$, tertile 2 : $1.57<\mathrm{TSH} \leq 2.59(n=277)$, and tertile $3: \mathrm{TSH}>2.59(n=259)$. Data are presented as the mean \pm standard deviation.

is usually slight and reversible, and would normalize with the achievement of euthyroid status [5-9], which suggested thyrotoxemia, at least in part, is related to $\mathrm{PH}$.

As for the pathogenetic mechanisms of $\mathrm{PH}$ under the background of hyperthyroidism and increased thyroid hormones levels, there are several hypotheses. Firstly, it was demonstrated that thyroid hormones may exert a direct action on pulmonary endothelial and smooth muscle cells [24-27]. Huesseini et al. reported that subtotal thyroidectomy reversed the development of severe experimental $\mathrm{PH}$ in SuHx rats, and the $\mathrm{PH}$ phenotype could be restored after a supplement of thyroxine. And they also showed that thyroid hormones promote endothelial cell proliferation through extracellular signal-regulated protein kinase 1 and 2 phosphorylation, and the expression of $\alpha_{\mathrm{v}} \beta_{3}$ integrin, fibroblast growth factor- (FGF-) 2, and FGF receptor. Lai et al. indicated that propylthiouracil treatment could suppress the smooth muscle cells via gamma-secretase-mediated Notch3 signaling pathway, especially presenilin enhancer 2 (Pen-2) subunit, resulted in attenuated experimental PH. Secondly, thyroid hormones also have many effects on the heart. Even in euthyroid patients, thyroid hormone levels are associated with heart rate and cardiac structure and function [28]. As a result, increased hemodynamic stress could promote an increase in endothelial shear stress of the pulmonary arteries, followed by endothelial dysfunction [29], which might be an important mechanism for the onset of PH. Thirdly, thyroid hormones enhanced catecholamine sensitivity and decreased cholinergic tone, resulting in pulmonary vasoconstriction. High levels of thyroid hormones also lead to increased metabolism of the intrinsic pulmonary vasodilating substances (prostacyclin, nitric oxide) and decreased metabolism of the vasoconstrictors (serotonin, endothelin 1 , and thromboxane). The net effects of these processes contributed to an increase in pulmonary vascular resistance [30].

Former studies explored the association of thyroid hormones and $\mathrm{PH}$ predominantly in patients with hyperthyroidism, and the results were controversial. In an observational study by Marvisi et al. [10], correlations between PASP with both TSH and $\mathrm{FT}_{4}$ levels were observed in newly diagnosed hyperthyroidism patients without antithyroid treatment. Another study [11] recruited 50 consecutive patients with Graves' disease. No significant difference of $\mathrm{FT}_{3}, \mathrm{FT}_{4}$, or $\mathrm{TSH}$ levels was found between the $\mathrm{PH}$ and non-PH groups, and PASP was not related to $\mathrm{FT}_{3}$ or $\mathrm{FT}_{4}$ levels.

Although more and more human studies confirmed the relationship between $\mathrm{PH}$ and hyperthyroidism, whether thyroid hormones in the normal range affect the cardiovascular system still lacks evidence. Our study demonstrated that serum $\mathrm{FT}_{4}$ levels were independently associated with $\mathrm{PH}$ and PASP in euthyroid CAD patients, which suggested that $\mathrm{FT}_{4}$ in the normal range might play a role in the development of $\mathrm{PH}$. However, only one previous study investigated the association between $\mathrm{PH}$ with $\mathrm{FT}_{3}$ and $\mathrm{FT}_{4}$ in euthyroid patients. Ciccone et al. [12] enrolled 93 euthyroid patients, 70 suffering from Hashimoto's thyroiditis and 23 controls. They demonstrated PASP were not independently related to $\mathrm{FT}_{3}, \mathrm{FT}_{4}$, or $\mathrm{TSH}$ in this population. The discrepancy of the two studies might be due to the different study populations. Our study focused on 811 CAD patients with mean age 63.3 years old, and among them, $77.2 \%$ were males. Their study was conducted in female patients with Hashimoto's thyroiditis who were older than 18 years old, and their sample size was much smaller than ours. Further studies are needed to confirm the association of thyroid hormones with $\mathrm{PH}$ and PASP in euthyroid subjects.

It is widely accepted that the major effects of thyroid hormones on the cardiovascular system are exerted by triiodothyronine $\left(\mathrm{T}_{3}\right)$, which was mainly converted from the inactive form, thyroxine $\left(\mathrm{T}_{4}\right)$, in the peripheral tissues. Considering this conversion process, $\mathrm{FT}_{3}$ was supposed to have a similar effect on $\mathrm{PH}$ as $\mathrm{FT}_{4}$ did. However, we did not find any relationship between PASP and $\mathrm{FT}_{3}$. Compared with that of $\mathrm{FT}_{4}$, the reference range of $\mathrm{FT}_{3}$ for euthyroid status was relatively narrower; and therefore, it might be underpowered to detect a statistic significant 
TABLE 4: Multiple logistic regression analysis for pulmonary hypertension.

\begin{tabular}{|c|c|c|c|}
\hline Independent variables & $\begin{array}{c}\text { Model } 1 \\
\text { OR (95\% CI) }\end{array}$ & $\begin{array}{c}\text { Model } 2 \\
\text { OR }(95 \% \text { CI }) \\
\end{array}$ & $\begin{array}{c}\text { Model } 3 \\
\text { OR (95\% CI) }\end{array}$ \\
\hline Gender & $0.56(0.31-0.99)$ & $0.56(0.32-0.99)$ & $0.58(0.33-1.02)$ \\
\hline Age & $1.06(1.03-1.09)$ & $1.06(1.03-1.09)$ & $1.06(1.03-1.09)$ \\
\hline BMI & $1.01(0.94-1.10)$ & $1.02(0.94-1.11)$ & $1.02(0.94-1.10)$ \\
\hline SBP & $0.99(0.97-1.01)$ & $0.99(0.97-1.01)$ & $0.99(0.97-1.01)$ \\
\hline Smoking & $2.21(1.09-4.46)$ & $2.24(1.11-4.55)$ & $2.19(1.08-4.44)$ \\
\hline Hypertension & $1.74(0.93-3.28)$ & $1.64(0.87-3.10)$ & $1.73(0.92-3.25)$ \\
\hline ACEI \& ARB & $0.96(0.57-1.60)$ & $0.93(0.55-1.56)$ & $0.94(0.56-1.56)$ \\
\hline $\mathrm{CCB}$ & $0.78(0.44-1.41)$ & $0.76(0.42-1.37)$ & $0.78(0.43-1.40)$ \\
\hline Nitrates & $1.08(0.66-1.77)$ & $1.07(0.65-1.76)$ & $1.07(0.65-1.75)$ \\
\hline LVEF & $0.95(0.93-0.97)$ & $0.95(0.93-0.97)$ & $0.95(0.93-0.97)$ \\
\hline $\mathrm{FT}_{3}$ & $1.18(0.79-1.82)$ & l & l \\
\hline $\mathrm{FT}_{4}$ & I & $1.12(1.01-1.25)$ & I \\
\hline TSH & l & 1 & $0.97(0.84-1.12)$ \\
\hline
\end{tabular}

PH: pulmonary hypertension; BMI: body mass index; SBP: systolic blood pressure; $\mathrm{FT}_{4}$ : free thyroxine; $\mathrm{FT}_{3}$ : free triiodothyronine; $\mathrm{TSH}_{\text {: thyroid-stimulating }}$ hormone; LVEF: left ventricular ejection fraction; CCB: calcium channel blockers; ACEI \& ARB: ACE inhibitors and angiotensin II receptor antagonists. Independent variables included in the original model are as follows: model 1: $\mathrm{FT}_{3}$, gender, age, BMI, smoking, LVEF, SBP, history of hypertension, CCB, $\mathrm{ACE} \& \mathrm{ARB}$, and nitrates; model 2: $\mathrm{FT}_{4}$, gender, age, BMI, smoking, LVEF, SBP, history of hypertension, CCB, ACE \& ARB, and nitrates; and model 3: TSH, gender, age, BMI, smoking, LVEF, SBP, history of hypertension, CCB, ACE \& ARB, and nitrates.

TABLE 5: Multiple linear regression analysis for pulmonary artery systolic pressure.

\begin{tabular}{lccc}
\hline Independent & Model 1 & Model 2 & Model 3 \\
variables & $\beta \pm \mathrm{SE}$ & $\beta \pm \mathrm{SE}$ & $\beta \pm \mathrm{SE}$ \\
\hline Gender & $-0.32 \pm 0.67$ & $-0.58 \pm 0.65$ & $-0.49 \pm 0.65$ \\
Age & $0.10 \pm 0.03^{*}$ & $0.11 \pm 0.03^{*}$ & $0.11 \pm 0.03^{*}$ \\
$\mathrm{BMI}$ & $0.03 \pm 0.09$ & $0.05 \pm 0.09$ & $0.03 \pm 0.09$ \\
SBP & $-0.02 \pm 0.02$ & $-0.02 \pm 0.02$ & $-0.02 \pm 0.02$ \\
Smoking & $1.45 \pm 1.00$ & $1.39 \pm 0.99$ & $1.42 \pm 1.00$ \\
Hypertension & $0.34 \pm 0.67$ & $0.32 \pm 0.67$ & $0.38 \pm 0.67$ \\
ACEI \& ARB & $0.08 \pm 0.57$ & $0.07 \pm 0.57$ & $0.08 \pm 0.57$ \\
CCB & $-0.45 \pm 0.67$ & $-0.49 \pm 0.67$ & $-0.43 \pm 0.67$ \\
Nitrates & $-0.30 \pm 0.55$ & $-0.31 \pm 0.55$ & $-0.27 \pm 0.55$ \\
LVEF & $-0.14 \pm 0.03^{*}$ & $-0.13 \pm 0.03^{*}$ & $-0.14 \pm 0.03^{*}$ \\
$\mathrm{FT}_{3}$ & $-0.53 \pm 0.49$ & $/$ & $/$ \\
$\mathrm{FT}_{4}$ & $/$ & $0.27 \pm 0.12^{*}$ & $/$ \\
TSH & $/$ & $/$ & $0.00 \pm 0.15$ \\
\hline
\end{tabular}

PASP: pulmonary artery systolic pressure; BMI: body mass index; SBP: systolic blood pressure; $\mathrm{FT}_{4}$ : free thyroxine; $\mathrm{FT}_{3}$ : free triiodothyronine; TSH: thyroid-stimulating hormone; LVEF: left ventricular ejection fraction; CCB: calcium channel blockers; ACEI \& ARB: ACE inhibitors and angiotensin II receptor antagonists. Independent variables included in the original model are as follows: model 1: $\mathrm{FT}_{3}$, gender, age, BMI, smoking, LVEF, SBP, history of hypertension, CCB, ACE \& ARB, and nitrates; model 2: $\mathrm{FT}_{4}$, gender, age, BMI, smoking, LVEF, SBP, history of hypertension, $\mathrm{CCB}, \mathrm{ACE} \& \mathrm{ARB}$, and nitrates; and model 3: TSH, gender, age, BMI, smoking, LVEF, SBP, history of hypertension, CCB, ACE \& $\mathrm{ARB}$, and nitrates. ${ }^{*} P<0.05$.

association between $\mathrm{FT}_{3}$ and PASP. In addition, although there is a regulatory negative feedback loop between TSH and thyroid hormones, the correlations between TSH and $\mathrm{FT}_{4}$ and $\mathrm{FT}_{3}$ were relatively low in our study $(\beta=-0.17$ and $\beta=-0.14$, resp.). As a result, our study did not find that TSH was associated with PASP. Sahin et al. [13] recruited 30 consecutive euthyroid patients with Hashimoto's thyroiditis and 30 healthy controls and found that correlation between PASP and TSH was not significant, which was consistent with our observations.

Besides, our results showed that age and smoking were independent risk factors for $\mathrm{PH}$. According to previous studies, advanced age and a greater tobacco exposure were associated with severely reduced diffusing capacity of the lung for carbon monoxide in $\mathrm{PH}$ patients [31]. Tobacco smoke exposure can lead to an elevation of pulmonary artery pressure, long before destruction of the lung parenchyma [32-34]. Keusch et al. observed a significantly higher smoking prevalence in $\mathrm{PH}$ men and significantly more exposure to tobacco smoke in PH women to in a case-control study [35]. Another study in hemodialysis patients also found that $\mathrm{PH}$ was related to cigarette smoking [36]. In the Rotterdam Study [37], the mean age of their study subjects was 76.4 years which was similar to our population. The prevalence of $\mathrm{PH}$ was higher in older participants compared to that in younger ones, and older age was independently associated with higher PASP, which was in agreement with our findings. And the association of age with $\mathrm{PH}$ was also observed in other studies [38-41].

Our study has several limitations. First, since this was a retrospective study, the causative relationship between $\mathrm{FT}_{4}$ and PASP cannot be determined. And lacking of data of some patients may contribute to statistic bias in our analysis. Prospective studies are needed to confirm our results in the future. Second, the use of medical records might be a further limitation that memory bias and reporter bias may exist. However, we also completed a structured interview for each subject in order to minimize the possible bias. Third, PASP were measured by echocardiography instead of right heart 
catheterization. Although right heart catheterization is still the gold standard technique, echocardiography has been proven to be reliable for the detection of $\mathrm{PH}$ [42-46]. Fourth, serum thyroid autoantibody levels have not been evaluated, so that we cannot exclude the effects of autoimmunity when analyzing the association between thyroid hormones and PASP.

In conclusion, our study demonstrated that in euthyroid patients with $\mathrm{CAD}, \mathrm{FT}_{4}$ was an independent risk factor for $\mathrm{PH}$, and $\mathrm{FT}_{4}$ levels were independently associated with PASP.

\section{Ethical Approval}

All procedures performed in studies involving human participants were in accordance with the ethical standards of the institutional and/or national research committee and with the 1964 Helsinki Declaration and its later amendments or comparable ethical standards.

\section{Disclosure}

None of the authors have any potential conflicts of interest associated with this research.

\section{Conflicts of Interest}

The authors declare that they have no conflicts of interest.

\section{Authors' Contributions}

Yan Ling and Xin Gao contributed equally to this work.

\section{Acknowledgments}

The authors would like to thank Dr. Junbo Ge, Dr. Juying Qian, Dr. Daxin Zhou, Dr. Jingmin Zhou, and Dr. Yangang $\mathrm{Su}$, Department of Cardiology, Zhongshan Hospital, Fudan University, for their cooperation and help in this study. This study was supported by the following grants: the National Key Clinical Specialty Construction Project of the Ministry of Health in 2011 (Health Office of Medical Affairs letter (2011) no. 873) and the National Natural Science Foundation of China (Grant no. 81402207 to Yan Ling).

\section{References}

[1] D. B. Badesch, K. M. Wynne, S. Bonvallet, N. F. Voelkel, C. Ridgway, and B. M. Groves, "Hypothyroidism and primary pulmonary hypertension: an autoimmune pathogenetic link?" Annals of Internal Medicine, vol. 119, no. 1, pp. 44-46, 1993.

[2] A. L. Curnock, R. A. Dweik, B. H. Higgins, B. H. Higgins, H. F. Saadi, and A. C. Arroliga, "High prevalence of hypothyroidism in patients with primary pulmonary hypertension," The American Journal of the Medical Sciences, vol. 318, no. 5, pp. 289-292, 1999.

[3] J. W. Chu, P. N. Kao, J. L. Faul, and R. L. Doyle, "High prevalence of autoimmune thyroid disease in pulmonary arterial hypertension," Chest, vol. 122, no. 5, pp. 1668 1673, 2002.

[4] J. H. Li, R. E. Safford, J. F. Aduen, M. G. Heckman, J. E. Crook, and C. D. Burger, "Pulmonary hypertension and thyroid disease," Chest, vol. 132, no. 3, pp. 793-797, 2007.

[5] M. Marvisi, P. Zambrelli, M. Brianti, G. Civardi, R. Lampugnani, and R. Delsignore, "Pulmonary hypertension is frequent in hyperthyroidism and normalizes after therapy," European Journal of Internal Medicine, vol. 17, no. 4, pp. 267-271, 2006.

[6] M. Armigliato, R. Paolini, S. Aggio et al., "Hyperthyroidism as a cause of pulmonary arterial hypertension: a prospective study," Angiology, vol. 57, no. 5, pp. 600-606, 2006.

[7] A. Yazar, O. Doven, S. Atis et al., "Systolic pulmonary artery pressure and serum uric acid levels in patients with hyperthyroidism," Archives of Medical Research, vol. 34, no. 1, pp. 35-40, 2003.

[8] J. Merce, S. Ferras, C. Oltra et al., "Cardiovascular abnormalities in hyperthyroidism: a prospective Doppler echocardiographic study," The American Journal of Medicine, vol. 118, no. 2, pp. 126-131, 2005.

[9] C. W. Siu, X. H. Zhang, C. YUNG, A. W. Kung, C. P. Lau, and H. F. Tse, "Hemodynamic changes in hyperthyroidism-related pulmonary hypertension: a prospective echocardiographic study," The Journal of Clinical Endocrinology and Metabolism, vol. 92, no. 5, pp. 1736-1742, 2007.

[10] M. Marvisi, M. Brianti, G. Marani, R. Del Borello, M. L. Bortesi, and A. Guariglia, "Hyperthyroidism and pulmonary hypertension," Respiratory Medicine, vol. 96, no. 4, pp. 215220, 2002.

[11] T. Sugiura, S. Yamanaka, H. Takeuchi, N. Morimoto, M. Kamioka, and Y. Matsumura, "Autoimmunity and pulmonary hypertension in patients with Graves' disease," Heart and Vessels, vol. 30, no. 5, pp. 642-646, 2015.

[12] M. M. Ciccone, A. Zito, A. Ciampolillo et al., "Pulmonary hypertension and Hashimoto's thyroiditis: does a relationship exist?” Endocrine, vol. 48, no. 2, pp. 621-628, 2015.

[13] M. Sahin, L. E. Sade, N. B. Tutuncu et al., "Systolic pulmonary artery pressure and echocardiographic measurements in patients with euthyroid Hashimoto's thyroiditis," Journal of Endocrinological Investigation, vol. 32, no. 6, pp. 530532, 2009.

[14] D. A. Fullerton, S. D. Jones, F. L. Grover, and M. I. RC Jr, "Adenosine effectively controls pulmonary hypertension after cardiac operations," The Annals of Thoracic Surgery, vol. 61, no. 4, pp. 1118-1123, 1996, discussion 1123-1114.

[15] J. E. Moller, G. S. Hillis, J. K. Oh, and P. A. Pellikka, "Prognostic importance of secondary pulmonary hypertension after acute myocardial infarction," The American Journal of Cardiology, vol. 96, no. 2, pp. 199-203, 2005.

[16] B. RISTOW, S. ALI, X. REN, M. A. Whooley, and N. B. Schiller, "Elevated pulmonary artery pressure by Doppler echocardiography predicts hospitalization for heart failure and mortality in ambulatory stable coronary artery disease: the Heart and Soul Study," Journal of the American College of Cardiology, vol. 49, no. 1, pp. 43-49, 2007.

[17] L. G. Rudski, W. W. Lai, J. Afilalo et al., "Guidelines for the echocardiographic assessment of the right heart in adults: a report from the American Society of Echocardiography endorsed by the European Association of Echocardiography, a registered branch of the European Society of Cardiology, 
and the Canadian Society of Echocardiography," Journal of the American Society of Echocardiography, vol. 23, no. 7, pp. 685713, 2010, quiz 786-688.

[18] D. Kong, X. Shu, L. Dong et al., "Right ventricular regional systolic function and dyssynchrony in patients with pulmonary hypertension evaluated by three-dimensional echocardiography," Journal of the American Society of Echocardiography, vol. 26, no. 6, pp. 649-656, 2013.

[19] G. Simonneau, M. A. Gatzoulis, I. Adatia et al., "Updated clinical classification of pulmonary hypertension," Journal of the American College of Cardiology, vol. 62, 25 Supplement, pp. D34-D41, 2013.

[20] K. K. Yeo, T. M. Maddox, E. Carey, R. I. Low, and K. A. Shunk, "Right- and left-sided heart catheterization as a quality marker for catheterization laboratories (from the national veterans affairs clinical assessment reporting and tracking program)," The American Journal of Cardiology, vol. 114, no. 11, pp. 1758-1762, 2014.

[21] Y. Zhang, B. K. Kim, Y. Chang et al., "Thyroid hormones and coronary artery calcification in euthyroid men and women," Arteriosclerosis, Thrombosis, and Vascular Biology, vol. 34, no. 9, pp. 2128-2134, 2014.

[22] E. S. Kim, J. A. SHIN, J. Y. SHIN et al., “Association between low serum free thyroxine concentrations and coronary artery calcification in healthy euthyroid subjects," Thyroid, vol. 22, no. 9, pp. 870-876, 2012.

[23] F. Ertas, H. Kaya, and M. S. Soydinc, "Low serum free triiodothyronine levels are associated with the presence and severity of coronary artery disease in the euthyroid patients: an observational study," Anadolu Kardiyoloji Dergisi, vol. 12, no. 7, pp. 591-596, 2012.

[24] T. Kasahara, K. Tsunekawa, K. Seki, M. Mori, and M. Murakami, "Regulation of iodothyronine deiodinase and roles of thyroid hormones in human coronary artery smooth muscle cells," Atherosclerosis, vol. 186, no. 1, pp. 207-214, 2006.

[25] A. Al Husseini, G. Bagnato, L. Farkas et al., "Thyroid hormone is highly permissive in angioproliferative pulmonary hypertension in rats," The European Respiratory Journal, vol. 41, no. 1, pp. 104-114, 2013.

[26] Y. J. Lai, G. J. Chang, Y. H. Yeh, J. H. Pang, C. C. Huang, and W. J. Chen, "Propylthiouracil attenuates experimental pulmonary hypertension via suppression of pen-2, a key component of gamma-secretase," PloS One, vol. 10, no. 9, article e0137426, 2015.

[27] F. B. Davis, S. A. Mousa, L. O'Connor et al., "Proangiogenic action of thyroid hormone is fibroblast growth factordependent and is initiated at the cell surface," Circulation Research, vol. 94, no. 11, pp. 1500-1506, 2004.

[28] G. L. Roef, Y. E. Taes, J. M. Kaufman et al., "Thyroid hormone levels within reference range are associated with heart rate, cardiac structure, and function in middle-aged men and women," Thyroid, vol. 23, no. 8, pp. 947-954, 2013.

[29] V. O. Kheyfets, L. Rios, T. Smith et al., "Patient-specific computational modeling of blood flow in the pulmonary arterial circulation," Computer Methods and Programs in Biomedicine, vol. 120, no. 2, pp. 88-101, 2015.

[30] D. R. Silva, M. B. Gazzana, A. B. John, D. R. Siqueira, A. L. Maia, and S. S. Barreto, "Pulmonary arterial hypertension and thyroid disease," Jornal Brasileiro de Pneumologia, vol. 35, no. 2, pp. 179-185, 2009.
[31] P. Trip, E. J. Nossent, F. S. De Man et al., "Severely reduced diffusion capacity in idiopathic pulmonary arterial hypertension: patient characteristics and treatment responses," The European Respiratory Journal, vol. 42, no. 6, pp. 15751585, 2013.

[32] S. Santos, V. I. Peinado, J. Ramirez et al., "Characterization of pulmonary vascular remodelling in smokers and patients with mild COPD," The European Respiratory Journal, vol. 19, no. 4, pp. 632-638, 2002.

[33] K. A. Hale, S. L. Ewing, B. A. Gosnell, and D. E. Niewoehner, "Lung disease in long-term cigarette smokers with and without chronic air-flow obstruction," The American Review of Respiratory Disease, vol. 130, no. 5, pp. 716-721, 1984.

[34] V. I. Peinado, J. A. Barbera, P. Abate et al., "Inflammatory reaction in pulmonary muscular arteries of patients with mild chronic obstructive pulmonary disease," American Journal of Respiratory and Critical Care Medicine, vol. 159, no. 5, Part 1, pp. 1605-1611, 1999.

[35] S. Keusch, F. F. Hildenbrand, T. Bollmann et al., "Tobacco smoke exposure in pulmonary arterial and thromboembolic pulmonary hypertension," Respiration, vol. 88, no. 1, pp. 3845, 2014.

[36] C. E. Dagli, H. Sayarlioglu, E. Dogan et al., "Prevalence of and factors affecting pulmonary hypertension in hemodialysis patients," Respiration, vol. 78, no. 4, pp. 411-415, 2009.

[37] E. M. Moreira, H. Gall, M. J. Leening et al., "Prevalence of pulmonary hypertension in the general population: the Rotterdam Study," PloS One, vol. 10, no. 6, article e0130072, 2015.

[38] V. Fayngersh, F. Drakopanagiotakis, M. F. Dennis, and J. R. Klinger, "Pulmonary hypertension in a stable communitybased COPD population," Lung, vol. 189, no. 5, pp. 377-382, 2011.

[39] C. S. Lam, B. A. Borlaug, G. C. Kane, F. T. Enders, R. J. Rodeheffer, and M. M. Redfield, "Age-associated increases in pulmonary artery systolic pressure in the general population," Circulation, vol. 119, no. 20, pp. 2663-2670, 2009.

[40] B. M. Mcquillan, M. H. Picard, M. Leavitt, and A. E. Weyman, "Clinical correlates and reference intervals for pulmonary artery systolic pressure among echocardiographically normal subjects," Circulation, vol. 104, no. 23, pp. 2797-2802, 2001.

[41] A. D'Andrea, R. Naeije, E. Grunig et al., "Echocardiography of the pulmonary circulation and right ventricular function: exploring the physiologic spectrum in 1,480 normal subjects," Chest, vol. 145, no. 5, pp. 1071-1078, 2014.

[42] T. Constantinescu, S. L. Magda, R. Niculescu et al., "New echocardiographic tehniques in pulmonary arterial hypertension vs. right heart catheterization - a pilot study," Maedica (Buchar), vol. 8, no. 2, pp. 116-123, 2013.

[43] J. A. Mazurek and P. R. Forfia, "Enhancing the accuracy of echocardiography in the diagnosis of pulmonary arterial hypertension: looking at the heart to learn about the lungs," Current Opinion in Pulmonary Medicine, vol. 19, no. 5, pp. 437-445, 2013.

[44] Y. Xie, B. M. Burke, A. Kopelnik et al., "Echocardiographic estimation of pulmonary vascular resistance in chronic thromboembolic pulmonary hypertension: utility of right heart Doppler measurements," Echocardiography, vol. 31, no. 1, pp. 29-33, 2014. 
[45] P. Lindqvist, S. Soderberg, M. C. Gonzalez, E. Tossavainen, and M. Y. Henein, "Echocardiography based estimation of pulmonary vascular resistance in patients with pulmonary hypertension: a simultaneous Doppler echocardiography and cardiac catheterization study," European Journal of Echocardiography, vol. 12, no. 12, pp. 961-966, 2011.

[46] M. D'Alto, E. Romeo, P. Argiento et al., "Accuracy and precision of echocardiography versus right heart catheterization for the assessment of pulmonary hypertension," International Journal of Cardiology, vol. 168, no. 4, pp. 4058-4062, 2013. 


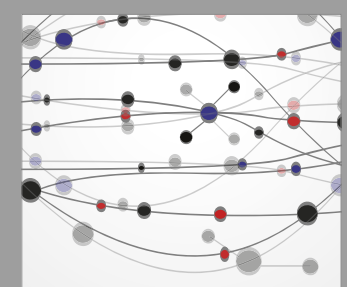

The Scientific World Journal
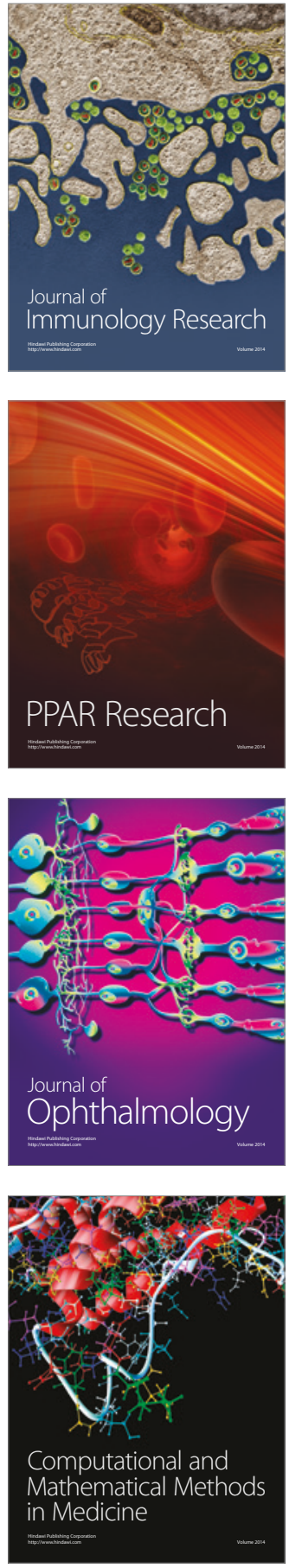

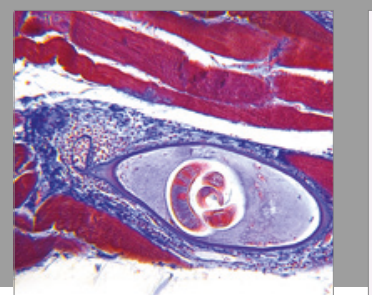

Gastroenterology Research and Practice
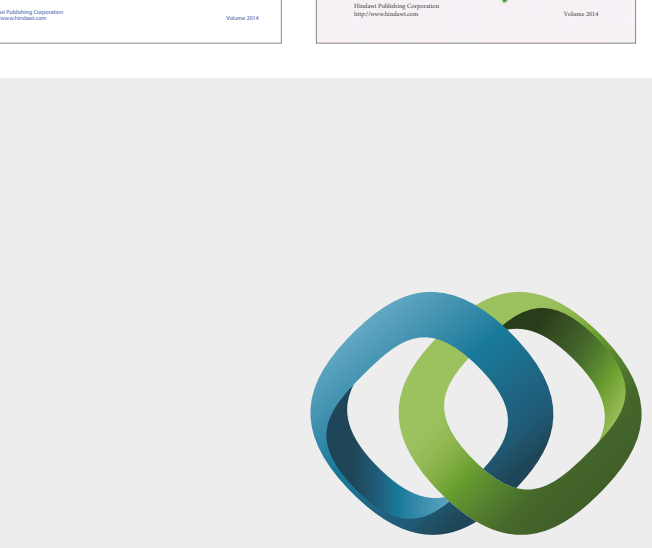

\section{Hindawi}

Submit your manuscripts at

https://www.hindawi.com
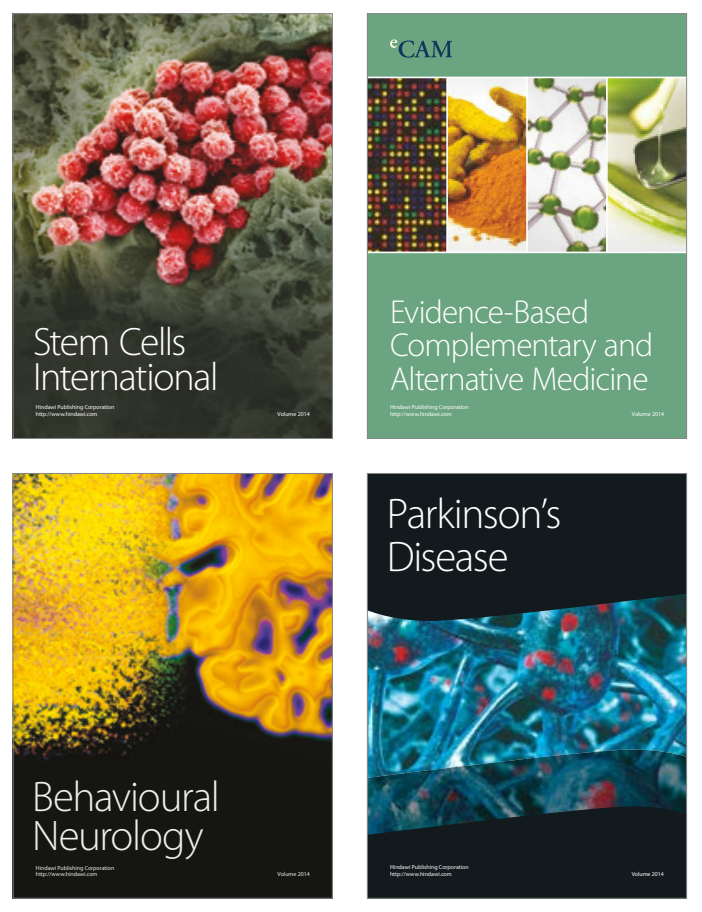
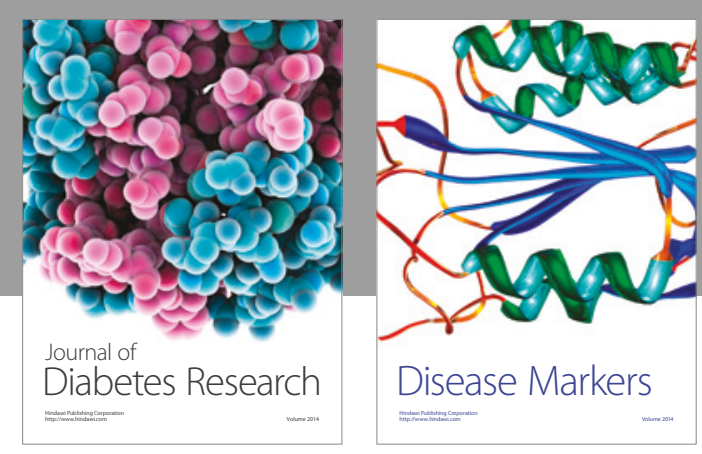

Disease Markers
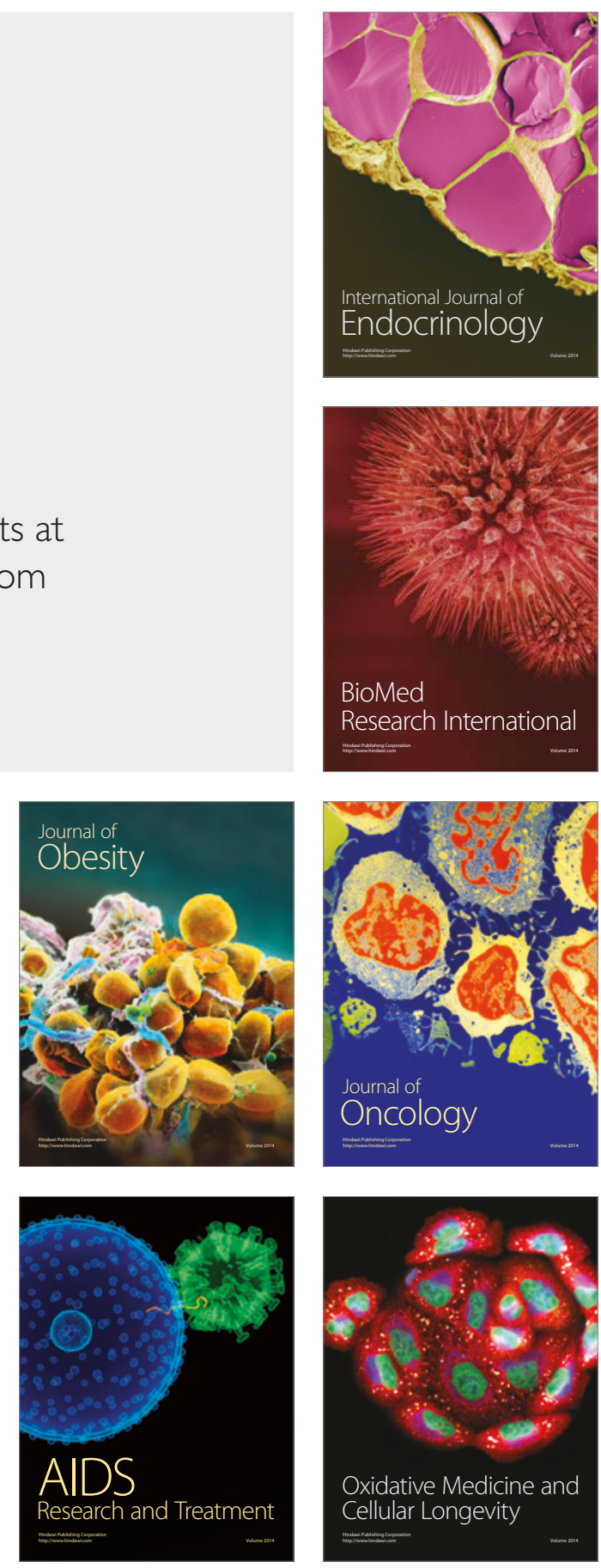